\title{
FINDINGS FROM AN ACCELERATED IN VIVO CORROSION MODEL OF MAGNESIUM
}

\author{
C. K. Yuen*, W. Y. Ip
}

Department of Orthopaedics and Traumatology, The University of Hong Kong

*: ckyuen2@gmail.com

Although first described in 1907 [1], absorbable metallic implant of magnesium (and its alloys) had not been a popular biomaterial, although some renewed interest arose recently.

In this short article, we are presenting some findings on its in vivo corrosion behavior, and no attempt is made to draw its medical significance.

Subcutaneous gas accumulation (after magnesium reacts with water) and uneven corrosion were commonly mentioned as its major limitations [2] in previous studies. The former causes unnecessary nuisance, while the latter causes the implant to lose mechanical integrity too quickly.

We have recently conducted two rounds of animal experiments with rods of magnesium and its alloys, by implanting them subcutaneously into the back of laboratory mice. The first round employed $99.95 \% \mathrm{Mg}$ and some $\mathrm{Mg}$ alloys for assessing their potential toxicity, and no intoxication was observed [3]. The second round employs an accelerated-corrosion model of $99.95 \%$ pure magnesium only, with other post-operative care in some groups of mice (which are irrelevant to the discussion here).

Side findings of the second animal model were the most interesting. Upon dissection on the mice, it was found that some gas bubbles occurred under the fibrous layer surrounding the implants. However, we have some unexpected findings: a) gas bubbles were occurring at all sides of the implant, not just the outer side; b) areas under the gas bubbles were badly oxidized, forming concave pits containing a white substance, presumably $\mathrm{MgO}$; and c) after the fibrous layer on the magnesium implant is removed, implant surface quickly oxidizes in air.

Actually, although Mg alloys in the first round corroded so slow that the gas should have been absorbed by the body; tiny gas bubbles were still observed under the peri-implant 
fibrous layer upon dissection after 6 months [3], indicating that the fibrous layer is not sufficiently permeable to gas.

Therefore, we speculate that uneven corrosion of magnesium and magnesium alloys in the body are caused by the presence of peri-implant gas bubbles, which creates an uneven corrosion environment on the implant surface.

The mainstream engineering approach to develop alloys with high in vitro corrosion resistance was already questioned by other researchers [4], and this study only adds one more clue to it. As gaseous evolution is inevitable in magnesium corrosion, we may need to develop new approaches to promote in vivo gas absorption to ensure more even corrosion of magnesium and magnesium alloys. Using anticoagulants to increase capillary permeability may be a possible method, but further discussion is beyond the scope of this article.

\section{References}

[1] G.B. Stroganov, E. Savitsky, T. Mikhailovich, et al. Magnesium-base alloys for use in bone surgery. (1972) US Patent no. 3,687,135

[2] M.P. Staiger, A.M. Pietak, J. Huadmai, G. Dias. Magnesium and its alloys as orthopedic biomaterials: A review. Biomaterials 27 (2006), pp.1728-34

[3] C.K. Yuen, W.Y. Ip. Biodegradable magnesium alloys for tissue engineering and other biological applications: unexpected findings from a mouse model. TERMIS EU 2008 Porto Meeting (2008), Poster presentation, abstract accepted [4] F. Witte, J. Fischer, J. Nellesen, H.-A. Crostack. In vitro and in vivo corrosion bmeasurements of magnesium alloys. Biomaterials 27 (2006), pp.1013-1018 